\title{
Multiple LGSs to correct conical anisokinetism
}

\author{
R. Ragazzoni ${ }^{1}$, S. Esposito ${ }^{2}$, and A. Riccardi ${ }^{2}$ \\ 1 Astronomical Observatory of Padova, vicolo dell'Osservatorio 5, I-35122 Padova, Italy \\ e-mail: ragazzoni@astrpd.pd.astro.it \\ 2 Arcetri Astrophysical Observatory, Largo Enrico Fermi 5, I-50125 Firenze, Italy \\ e-mail: esposito@arcetri.astro.it,riccardi@arcetri.astro.it
}

Received March 18; accepted July 31, 1997

\begin{abstract}
It is shown that, in the multiple LGS configuration proposed by Tallon \& Foy (1990) used for the correction of the conical anisoplanatism, one can derive the correction for the conical anisokinetism effect also, provided that absolute tilt sensing is performed for only one of the various LGSs projected on the sky.
\end{abstract}

Key words: atmospheric effects - techniques: miscellameous — telescopes

\section{Introduction}

Laser Guide Stars (LGSs) using Sodium mesospheric backscattering resonance as a reference for Adaptive Optics (Foy \& Labeyrie 1985; Happer et al. 1994) are becoming a realistic viable solution for diffraction limited, whole sky coverage at large ground-based telescope.

For large aperture telescopes conical anisoplanatism effects (Gardner et al. 1990) due to the finite height of the laser beacon, deteriorates the achievable Sthrel ratio of an LGS-based Adaptive Optics system.

The latter source of error can be corrected using multiple LGSs; this is achieved obtaining a sort of threedimensional tomography of the wavefront perturbation on the various perturbing layers (Tallon \& Foy 1990; TF90 hereafter).

The lack of absolute tilt reference provided by an LGS (Pilkington 1987) can be solved (or at least minimized) in a number of solutions (Belen'kii 1994, 1995, 1996; Foy et al. 1992, 1995; Lukin 1996; Ragazzoni 1996a,b, 1997; Ragazzoni et al. 1995; Ragazzoni \& Marchetti 1997; Rigaut \& Gendron 1992). The proposed techniques are affected by some technical (Marchetti \& Ragazzoni, 1997) or fundamental limitations depending upon the adopted scheme for the absolute tilt determination. Conical anisokinetism is recognized (Esposito et al.

Send offprint requests to: R. Ragazzoni
1996; Neymann 1996; Riccardi et al. 1996) as a fundamental limitation affecting any sort of LGS tilt correction other than the ones based solely on natural guide stars (the latter offering a sky coverage far from the $100 \%$ at optical wavelenghts). The effects due to the distribution of the turbulence with the altitude (Ragazzoni et al. 1997) are not taken in consideration here and will be the subject of a forthcoming paper.

The interested reader can consult the cited papers for details on conical anisokinetism. Here we recall that we proposed such a term to identify the tilt error due to the conical shape of the sampled atmosphere volume, provided the knowledge of the ground to layer tilt experienced by the laser beam. The effect can be as severe as to deteriorate the achievable Sthrel ratio of a factor $S \approx 0.25$ when a $D=8.0 \mathrm{~m}$ telescope is observing in the visible under average seeing conditions and assuming an Hufnagel-Valley $C_{n}^{2}(z)$ turbulence distribution model.

\section{Conditions on the multiple LGSs scheme}

We assume, in the following, that the technique described by TF90 is fully implemented. Of the various conditions to be met in order to have the applicability to their technique we briefly discuss the one strictly related to the problem under investigation in this paper. We refer to the assumption that the position of the various LGSs on the Sodium layer is known. Obviously the exact knowledge of the LGSs positions solve the absolute tilt determination problem. However it is to be pointed out that the position of the LGSs required in the TF90 paper is characterized by an uncertainty such that a ray from the laser beacon at an height of $H \approx 95 \mathrm{~km}$ to a given point on the telescope aperture can be located on each disturbing layer with an uncertainty much smaller than $r_{0}$.

Assuming the maximum height of substantially disturbing layer of the order of $h_{\max } \approx 10 \mathrm{~km}$ it is easy to see that this will translates into an angular displacement of the LGS beacon as seen from the ground of the order of: 
$\theta \approx \frac{r_{0}}{h_{\max }}$

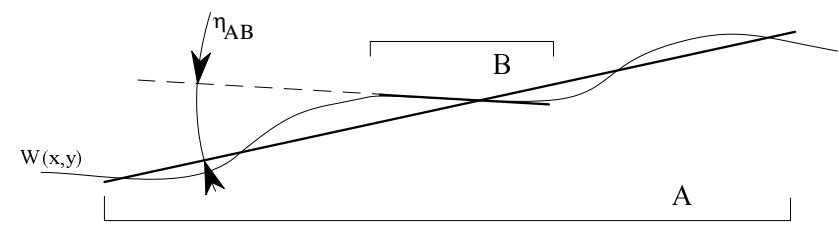

Fig. 1. In this unidimensional section of the wavefront $W(x, y)$ the relative tilt difference experienced by the wavefront in the domains $A$ and $B$ is denoted by $\eta_{A B}$. This quantity does not depends upon the absoulte tilt knowledge of $W$ in the whole domain

This angular displacement is to be compared with the rms fluctuation upward tilt angle of the LGS beacon given by the usual relationship (Acton 1995; Brandt et al. 1987; O'Byrne et al. 1995; Sarazin \& Roddier 1990):

$\sigma \approx 0.4 \frac{\lambda}{D_{\mathrm{p}}}\left(\frac{D_{\mathrm{p}}}{r_{0}}\right)^{5 / 6}$

where $D_{\mathrm{p}}$ is the laser projector aperture and $\lambda$ is the laser beacon wavelength. The ratio $\gamma=\sigma / \theta$ gives the goodness of the assumption made in TF90 regarding the LGS absolute position knowledge: $\gamma=0$ means that the knowledge of the position on the sodium layer is unaffected by the ground to layer propagation, while $\gamma=1$ means that the Sodium spot displacement due to upward tilt is great enough to make the rms shift of the light ray equal to $r_{0}$ on the higher layer. It can be turned out that such a quantity is given by:

$\gamma \approx 0.4 \frac{\lambda h_{\max }}{D^{1 / 6} r_{0}^{11 / 6}}$

With a projector aperture of $D=0.5 \mathrm{~m}$ and median seeing conditions of the order of $r_{0}=0.15 \mathrm{~m}$ a figure of $\gamma \approx 0.073$ is obtained.

This calculation shows that, while ground to layer perturbation is usually high enough to destroy any useful tilt information on the LGS, it is not so large to rule out one of the basic assumption made by TF90 in order to make the conical anisoplanatism correction a feasible technique.

\section{Conical anisokinetism error determination}

In the TF90 approach the overall tilt term can be retrieved if a suitable NGS is available in the field. In the present approach we show in the following that the knowledge of the absolute tilt of only one of the various LGS allow to obtain the absolute tilt free from the conical anisokinetism effect.

\subsection{Relative tilts within portions of the same wavefront}

Let us consider a wavefront distortion $W(x, y)$ introduced by a turbulence layer and defined in some domain of the $(x, y)$ plane at the height of the layer itself (see Fig. 1 for an unidimensional section). Let us also consider two subdomains of this plane denoted by the indexes $A$ and $B$. While the absolute tilt of $W$ can be unknown, the knowledge of the higher order shape of the wavefront enables us to define uniquely the relative tilt measured considering the wavefront portions $A$ and $B$. Such a quantity is denoted in the following by $\eta_{A B}$ and does not depends upon the absolute tilt of $W$. For each of the layers in the TF90 technique one can figure out any quantity of this type between single portions of the same wavefront. As a particular case (see Fig. 2) TF90 allows for the determination of the detailed wavefront shape for each layer as seen on the cylindrical shape beam going from the telescope entrance to the astronomical target. For any of the projected LGSs forming the constellation required to the proper turbulence tomography one can define as $\eta_{i}$ the relative tilt between the tilt generated by the $i$-th layer for the astronomical target and the tilt originated in the sub-domain defined by that LGS cone.

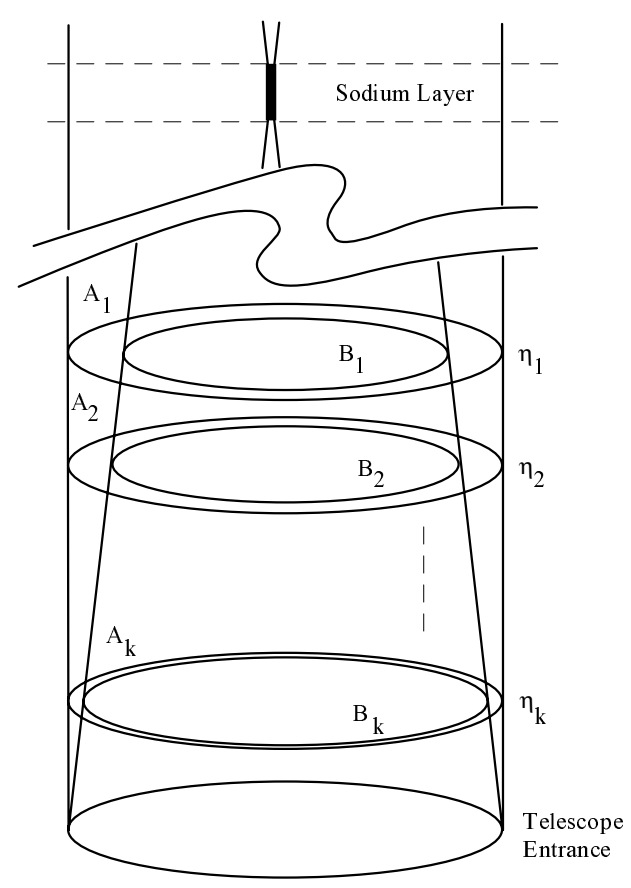

Fig. 2. For any $i$-th layer the quantity $\eta_{i}$ is defined as the differential tilt produced by that layer into the two domains $A$ related to an infinitely distant astronomical target and $B$ related to a particular LGS used for the TF90 tomographyc sensing

\subsection{Tilt tomography and conical-free tilt determination}

We assume that (for istance using any of the cited techniques) the absolute tilt of an LGS is sensed.

Let us define $\alpha_{i}^{\text {LGS }}$ as the angular tilt introduced by the sub-domain of the $i$-th layer intersected by the LGS 
beam. Because of the conical shape of the LGS beam, the optical path disturbance propagates from the layer to the telescope aperture with the same magnitude, but it is distributed on a larger surface. This effect reduces the angular LGS tilt contribution by a factor $\left(1-H_{i} / H^{\mathrm{LGS}}\right)$, where $H_{i}$ and $H^{\text {LGS }}$ are the heights of the layer and of the LGS respectively. This factor is given by the ratio between the diameter of the cone at the height $H_{i}$ and at the telescope level. The measured angular tilt of the LGS is given by:

$\alpha^{\mathrm{LGS}}=\sum_{i=1}^{k} \alpha_{i}^{\mathrm{LGS}}\left(1-\frac{H_{i}}{H^{\mathrm{LGS}}}\right)$.

Following Ragazzoni et al. (1997), with the use of Eq. (4) we define an effective tilting layer height $H^{\text {eff }}$ such that:

$\alpha^{\mathrm{LGS}}=\left(1-\frac{H^{\mathrm{eff}}}{H^{\mathrm{LGS}}}\right) \sum_{i=1}^{k} \alpha_{i}^{\mathrm{LGS}}$.

Equating the right sides of Eq. (4) and Eq. (5) one obtains:

$H^{\mathrm{eff}}=\frac{\sum_{i=1}^{k} H_{i} \alpha_{i}^{\mathrm{LGS}}}{\sum_{i=1}^{k} \alpha_{i}^{\mathrm{LGS}}}$.

On the other hand one can write the similar subdivision over the whole set of $k$ layers for the cylindrical shaped beam of an hypotethical Natural Guide Star (NGS):

$\alpha^{\mathrm{NGS}}=\sum_{i=1}^{k} \alpha_{i}^{\mathrm{NGS}}$.

The complete set of $\eta_{i}$, that are estimated through the TF90 technique, are given by:

$\eta_{i}=\alpha_{i}^{\mathrm{NGS}}-\alpha_{i}^{\mathrm{LGS}}$.

Summing over the whole $k$ layers the latter relationship, and using the Eq. (5), one can easily derive the following:

$\alpha^{\mathrm{NGS}}=\alpha^{\mathrm{LGS}}\left(1-\frac{H^{\mathrm{eff}}}{H^{\mathrm{LGS}}}\right)^{-1}-\sum_{i=1}^{k} \eta_{i}$

where the terms $\alpha^{\mathrm{LGS}}$ and $\sum_{i=1}^{k} \eta_{i}$ on the right side of Eq. (9) are experimentally measured, and the effective layer height $H^{\text {eff }}$ can be estimated as decribed in Ragazzoni et al. (1997). Care is to be given in a practical implementation to the effects of the error propagation from the estimation of the $\eta_{i}$ angles and the $H^{\text {eff }}$ parameter (this last figure, morevoer, can change with time). The proposed approach can be seen as an extension of the constraints imposed in the TF90 scheme. Their Eq. (13), in fact, imposes a dummy zero-tilt condition on the system to be solved to have the tomography of the turbulence layers. Subsituting such a constraint by the full set of Eq. (8) is an equivalent way to obtain the absolute tilt free from the conical anisokinetism error. As a further remark one should recall that for practical reasons one should limits the number of perturbing layer sensed by the TF90 technique and it is to be evaluated the impact of this approach to the overall tilt error budget. A detailed calculation and an estimation of the achievable Sthrel ratio is well beyond the limits of this paper and is not treated here.

\section{Conclusion}

Two fundamental aspects of LGS references in Adaptive Optics, namely the conical anisoplanatism and the tilt indetermination, have been attacked in the last years by several authors and using several approaches. It turns out that tilt recovery schemes are plagued by some apparently unavoidable conical anisokinetism error. We have shown in this paper that, one time the problem is faced in a global manner a simple solution to the conical anisokinetism error can be obtained.

\section{References}

Acton D.S., 1995, Appl. Opt. 34, 4526

Belen'kii M.S., 1994, SPIE Proc. 2201, 321

Belen'kii M.S., 1995, SPIE Proc. 2741, 289

Belen'kii M.S., 1996, SPIE Proc. 2956, 206

Brandt P.N., Mauter H.A., Smartt R., 1987, A\&A 188, 163

Esposito S., Riccardi A., Ragazzoni R., 1996, JOSA-A 13, 1916

Foy R., Boucher Y., Fleury B., Grynberg G., McCullough P.R., Migus A., Tallon M., 1992, ESO Proc. Conf. 42, 437

Foy R., Labeyrie A., 1985, A\&A 152, L29

Foy R., Migus A., Biraben F., Grynberg G., McCullough P.R., Tallon M., 1995, A\&AS 111, 569

Gardner C.S., Welsh B.M., Thompson L.A., 1990, Proc. IEEE 78,1721

Happer W., MacDonald G.J., Max C.E., Dyson F.J., 1994, JOSA-A 11, 263

Lukin I.P., 1996, OSA Technical Digest 13, Addendum AMB35

Marchetti E., Ragazzoni R., 1997, A\&AS 125, 551

Neymann C.R., 1996, Opt. Lett. 21, 1806

O'Byrne J.W., Bryant J.J., Minard R.A., Fekete P.W., Cram L.E., 1995, PASA 12, 106

Pilkington J.D.H., 1987, Nat 330, 116

Ragazzoni R., 1996a, A\&A 305, L13

Ragazzoni R., 1996b, ApJ 465, L73

Ragazzoni R., 1997, A\&A 319, L9

Ragazzoni R., Esposito S., Marchetti E., 1995, MNRAS 276, L76

Ragazzoni R., Marchetti E., 1997, SPIE Proc. 2871

Ragazzoni R., Marchetti E., Brusa G., 1997, A\&AS 121, 569

Riccardi A., Ragazzoni R., Esposito S., 1996, OSA Technical Digest 13, 84

Rigaut F., Gendron E., 1992, A\&A 261, 677

Sarazin M., Roddier F., 1990, A\&A 227, 294

Tallon M., Foy R., 1990, A\&A 235, 549 (TF90) 